\title{
Effects of Morinda citrifolia on Rheumatoid Arthritis in SKG Mice
}

\author{
Dyah Aninta Kustiarini, ${ }^{a, b}$ Toshiaki Nishigaki, ${ }^{c}$ Hiroyuki Kanno, ${ }^{c}$ and Hideto To ${ }^{*, a}$ \\ ${ }^{a}$ Department of Medical Pharmaceutics, Graduate School of Medicine and Pharmaceutical Science for Research, \\ University of Toyama; Toyama 930-0194, Japan: ${ }^{b}$ Centre for Agro-Based Industry; Bogor 16122, Indonesia: and \\ ${ }^{c}$ Department of Pathology, School of Medicine, Shinshu University; Nagano 390-8621, Japan. \\ Received June 24, 2018; accepted December 5, 2018
}

\begin{abstract}
Morinda citrifolia L., known as noni, originated from Indonesia exhibits various pharmacological activities including anti-inflammatory properties. However, the validity of noni fruit juice as a treatment for rheumatoid arthritis (RA), an autoimmune disorder, has not been confirmed yet. Therefore, the main purpose of this research was to evaluate the efficacy of noni fruit juice (INFJ) made in Indonesia using SKG mice as an animal model of RA, which shows the resembling characteristics of human RA patients. Furthermore, the safety of INFJ was examined by repeated dose experiments in mice. INFJ was mixed with water at $50 \%$ and administered to SKG mice sensitized with mannan, free access for 4 weeks. Arthritis scores of fore- and hind-leg joints were measured and the joints were histopathologically examined. The sub-acute and subchronic toxicities of INFJ were evaluated using BALB/c mice. The arthritic scores were significantly lower from the $\mathbf{7 d}$ after sensitization in the INFJ group than the control group. Histopathological examinations of the joints revealed inhibition of severity of RA. In both toxicity studies, INFJ did not show any toxicities. INFJ exhibited anti-arthritic activity in arthritic and histopathological examinations of the joints in SKG mice. Present study was the first report where noni juice may be effective against $R A$. The dose of noni juice showing efficacy against RA was confirmed safe from repeated dose studies in mice.
\end{abstract}

Key words rheumatoid; arthritis; noni; Morinda citrifolia; safety; SKG mouse

\section{INTRODUCTION}

Rheumatoid arthritis (RA) is a chronic autoimmune disorder that primarily affects the synovial membranes of multiple joints, resulting in damage to cartilage and bone around the joints. ${ }^{1-3)}$ Though RA occurs in $0.5-1.0 \%$ of the adult population worldwide and is common in females than males, ethnicity, residence or climate are not important factors in prevalence of RA. ${ }^{2,4} \mathrm{RA}$ is initiated by autoimmune $\mathrm{CD} 4^{+} \mathrm{T}$ cells because of the presence of autoantibodies, such as rheumatoid factors (RFs), and is related with particular haplotypes of the HLA-DR gene. ${ }^{4)}$

During the past few decades, many kinds of medicines such as disease-modifying antirheumatic drugs, biological agents, steroidal drugs, and non-steroidal anti-inflammatory drugs have been developed for RA. Unfortunately, there is still no effective medicinal treatment that has the ability to completely cure this disease. Moreover, some adverse effects such as infections and declined immune functions are reportedly caused by the long-term use of the drugs. Therefore, it was underway that researches to develop new synthetic medicines and assessment of the applicability of herbal natural medicines to the treatment of RA. ${ }^{5)}$

Morinda citrifolia L. (Rubiaceae), commonly known as noni is Indonesia-origin tropical herbal plant. $\left.{ }^{6}\right)$ Noni has a long history of use as a herbal remedy for various diseases in Indonesia where noni is commonly called as mengkudu or pace, and individual houses in rural regions grow at least one noni tree at their garden to prevent or cure diseases. From our knowledge, noni has been utilized as nutritional and medicinal fruit since the present Indonesian people were immigrated to Indonesia 6000 years ago and thereafter it was spread to Micronesian and Polynesian areas with boat immigration people because of its benefits for health and disease treatment. Noni fruit is one of the most important traditional medicinal products called Јати in Indonesia. In Indonesia, noni juice products are classified as semi-ethical medicine. Since noni fruit was reported its anti-cancerous action, ${ }^{7-9)}$ noni fruit has been popular and resumed as natural functional healthy food in the world. Anecdotal evidences demonstrate the biological activities of noni, including its potential anti-microbial, anti-cancer, anti-oxidant, anti-inflammatory, analgesic, hypotensive, antidiabetic, and cardiovascular activities. ${ }^{7-10)}$

The RA model of an animal is classified roughly into two models that develops arthritis by sensitizing collagen or Freund's adjuvant, and a model that occurs naturally. Arthritis of Complete Freund's Adjuvant (CFA) model was induced by injecting CFA-which is effective in stimulating cell-mediated immunity and leads to potentiation of $\mathrm{T}$ helper cells and also to the production of certain immunoglobulins and effector $\mathrm{T}$ cells. CFA model resembles arthritic in human by mimic clinical and pathological features of human RA such as swelling, bone erosion, cartilage degradation and loss of joint functions.

On the other hands, the SKG mouse is a BALB/c mouse that has a mutation on the gene encoding the Src homology 2 (SH2) domain of the zeta-associated protein of $70 \mathrm{kD}$ (ZAP-70), a key signal transduction molecule in $\mathrm{T}$ cells. This model spontaneously develops chronic autoimmune arthritis under conventional microbial conditions or following an injection of $\beta$-glucan-containing products (such as mannan) under specific-pathogen-free (SPF) conditions. Arthritis in SKG mice is characterized by symmetric affections in the small joints, elevations in interleukin (IL)-1, IL-6, tumor necrosis factor (TNF)- $\alpha$, and rheumatoid factor levels, joint destruction, and reduced bone density in addition to systemic manifestations including inflammation in the skin, blood vessels, 
and lungs, which resemble the clinical and histological characteristics of human RA. ${ }^{11-16)}$

Thus, both arthritis models cause similar joint changes. However, in order to develop potency of natural functional foods with new therapeutic substances and clarify their modes of action against human RA, autoimmune SKG mice model mimicked human chronic autoimmune arthritis is suitable rather than CFA model. This selection is based on the fact that SKG mice model as well as human RA spontaneously develop chronic autoimmune arthritis symptoms dominantly affected by genetic abnormalities.

In present study, we evaluated the antirheumatic effect of Indonesia-origin noni fruit juice (INFJ) using RA model animals, SKG mice. In addition, safety of INFJ was evaluated in sub-acute and sub-chronic toxicity studies using BALB/c mice.

\section{MATERIALS AND METHODS}

Animals SKG female mice (16-week-old) were purchased (CLEA, Japan) and used as an animal model of RA. BALB/c female mice (16-week-old) (SLC, Japan) were used for the toxicity tests. Both experimental animals were housed 3-6 mice per cage under 12-h light/dark cycle conditions (lights on and off at 7:00 and 19:00, respectively) at room temperature $\left(24 \pm 1{ }^{\circ} \mathrm{C}\right)$ and humidity $60 \pm 10 \%$. Experiments were performed after formal approval by the Institutional Ethical Committee for Research on Animals, Toyama University.

Preparation of Noni Juice INFJ used in all studies was provided by M\&K Laboratories Inc. (Matsumoto, Japan). INFJ was made from ripe noni fruits, freshly squeezed without the addition of water or preservatives, and produced under Hazard Analysis and Critical Control Pointsystem in Indonesia. In the present studies, INFJ was mixed with drinking water at concentration of $50 \%(\mathrm{v} / \mathrm{v})$.

Anti-arthritic Study SKG mice were divided into two groups. One group was given free access to 50\% INFJ for 4 weeks, and the control group was given free access to water only. The administration of INFJ began from $1 \mathrm{~d}$ before immunization. Mannan (Sigma-Aldrich, U.S.A.) was suspended in phosphate buffered saline (PBS) $(+)$ solution and injected intraperitoneally at a dose of $20 \mathrm{mg}$ to induce arthritis. Body weights and fluid intake of both groups were monitored 3 times weekly. Body weights were measured every day, and the fluid intakes were calculated as total intake per cage $(n=6)$ per a day.

Determination of Arthritic Scores The arthritic score of each mouse was evaluated by the sum of total score of wrist and ankle joints by referring to the arthritic scoring system developed by Sakuguchi et al. ${ }^{11)}$ Arthritis was graded on a scale of $0-1.0$ for each wrist/ankle: $0=$ no joint swelling; $0.1=$ swelling of one finger joint; $0.5=$ mild swelling of the wrist or ankle joint; $1.0=$ severe swelling of the wrist or ankle joint.

Histopathological Evaluation On day 28, SKG mice were sacrificed and the right and left wrist and ankle joints from 6 animals in each group were collected and fixed with $10 \%$ neutral fomalin for histopathological evaluation. Each joint was longitudinally cut and decalcified. After routine dehydration, the specimens were embedded in paraffin, cut into 5 - $\mu \mathrm{m}$-thick several sections, and stained with hematoxylineosin. Total 48 slides from all the wrist and ankle joints were examined under a light microscopy in a blind method.

Severity of the lesions in each joint was scored on 0-3 grades based on the histopathologically characteristic changes of RA and with reference to commonly used histological grading as follows. ${ }^{17)}$ Synoviocyte proliferation was graded on a scale of 0 to $3: 0=$ one cell layer; $1=2-3$ cell layers; $2=4-5$ cell layers; $3=$ more than 6 cell layers. Inflammation was graded on a scale of 0 to 3: $0=$ no inflammation; $1=$ slight inflammation; $2=$ moderate inflammation; $3=$ severe inflammation. Pannus formation was graded on a scale of 0 to 3 : $0=$ no formation; $1=$ localized pannus formation without the destruction of cartilage or bone; 2 = definite pannus formation with the partial destruction of cartilage and bone; $3=$ wide range in pannus formation and the destruction of cartilage and
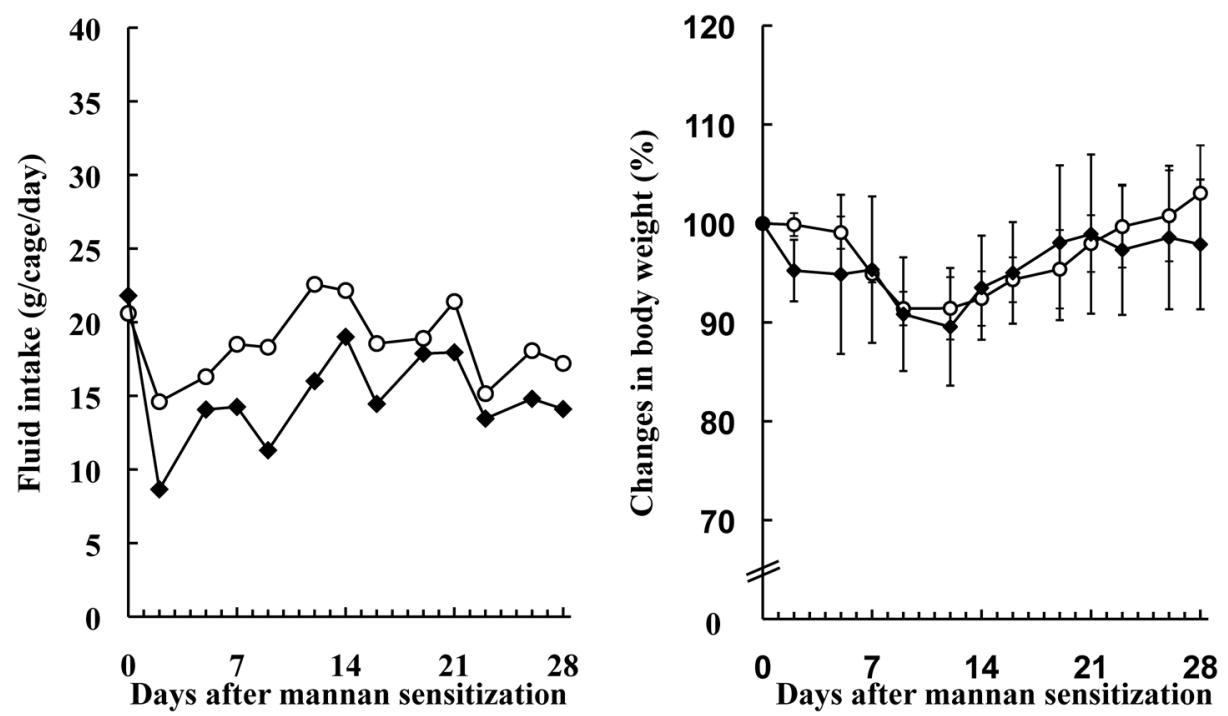

Fig. 1. Fluid Intake in SKG Mice (Left) and Changes in Body Weight in SKG Mice (Right)

SKG mice were given $50 \%(\mathrm{v} / \mathrm{v})$ INFJ (closed diamond) and the control group received drinking water (open circle). Each value of fluid intake represented fluid intake per cage in $1 \mathrm{~d}$ (each group contained 4 mice per cage), while each value of changes in body weight was the mean \pm S.D. $(n=4)$. 
bone.

The histopathology of each mouse was evaluated by the sum of all scores in the wrist and ankle joints.

Toxicity Studies BALB/c mice were divided into two groups, and were studied for 28 and $90 \mathrm{~d}$. One group was given free access to $50 \%$ INFJ, while the control group was served with water. After the end of each period of administration, blood samples were taken and then hematology was measured (Sysmex F-520, Japan). Serum were obtained to measure liver (Alanine transaminase (ALT), Transaminase CII Reagent Kit, Wako, Japan) and renal functions (Blood urea nitrogen (BUN), BUN Reagent Kit, Arbor Assays, U.S.A.).

Statistical Analyses Data were recorded as the mean \pm standard deviation (S.D.). Groups were compared by one-way ANOVA and differences between groups were analyzed using Student $t$-test. A probability level of less than $0.05 \%$ was evaluated to be significant.

\section{RESULTS}

INFJ Intake and Body Weight Fluid intake was lower in the INFJ group than in the control group throughout this study (Fig. 1, left). The lowest intake of INFJ was observed on day 2 after the mannan sensitization. On day 2, fluid intake per cage in one day was $14.6 \mathrm{~g}$ in the control group and $8.7 \mathrm{~g}$ in the INFJ group. Fluid intake was $40.4 \%$ lower in the INFJ group than in the control group on day 2. However, the decrease in fluid intake observed in the INFJ group did not lead to significant differences in body weights (Fig. 1, right).

Arthritic Score Significance differences were observed in arthritic scores between the two groups from days 7 to 28 after the mannan sensitization $(p<0.05$ or $p<0.01$, Fig. 2). On day 28, arthritic scores were $2.24 \pm 1.53$ (mean \pm S.D.) in the control group and $1.11 \pm 1.10$ in the INFJ group. The arthritic score was $50.4 \%$ lower in the INFJ group than in the control group, indicating INFJ prevented progression of RA.

Histopathology Score In the control group, typical changes were observed in multi-layered proliferation of synoviocytes with mononuclear and neutrophils infitration, pannus formation and nutrophils-rich exudates in the joint cavity (Figs. 3, A, B), but not severely in the INFJ group (Figs. 3, C, D).

The histopathology scores were significantly smaller in the INFJ group than the control group, particularly for inflammatory cell infiltration and pannus formation, and the statistical analysis of total scores revealed significantly smaller scores in the INFJ group (Table $1, p<0.05$ ).

Safety of Indonesian INFJ Parameters of safety of INFJ did not demonstrated any toxic findings in hematology and hepatic and renal functions, though BUN was higher in the INFJ group than the control group in 28-d administration study. However, BUN value was within normal range and no change was found in 90-d study (Tables 2, 3).

\section{DISCUSSION}

In order to evaluate anti-arthritic therepeutic effects of medicines, it has been common to use artificially induced arthritis such as type II collagen-induced arthritis. However, artificially induced arthritis is limited to examine the modes of naturally occurring arthritis in human because of non-phys- iological conditions. Recently, there are some animal models of RA developed naturally. One of them is SKG mice. SKG mice, a mutant of the gene encoding ZAP-70 on the BALB/c strain mice, spontaneously develop IL-17-secereting CD4 + T cells (Th17 cells)-mediated autoimmune arthritis clinically and immunologically resembling human RA, ${ }^{11)}$ and arthritis is induced by activation of complements. C5a is the key complement to promote arthritogenic T cells and their Th17 cell differentiation in arthritic joints of mannan-treated SKG mice under SPF animal environment. ${ }^{16)}$ In the present study, mannan was injected to evoke SKG arthritis. The arthritis shows joint swelling and characterized by subsynovial inflammatory cell infiltration, proliferation of synoviocytes, and pannus formation. They closely resemble the joint pathological findings of human RA. ${ }^{11)}$

Noni is one of Indonesia-origin tropical herbal plants and its fruit juice is prescribed as effective medicine for RA and several studies have confirmed the anti-inflammatory effects of INFJ. ${ }^{18-22)}$ The effect of INFJ for RA was evaluated using CFA induced arthritis rat. ${ }^{23)}$ However, there are no reports to evaluate the potential of noni fruit against RA resembling to human RA.

INFJ diluted to $50 \%$ was used. From preliminary studies, we decided to give INFJ by free access, but not gavage oral administration because the animals must not be exposed to stress by daily gavage dosing. Due to characteristic of INFJ with particle segments, $100 \%$ INFJ did not pass through a nozzle of water bottle. Finally we decided to apply at $50 \%$ INFJ which was the highest concentration for the mice to

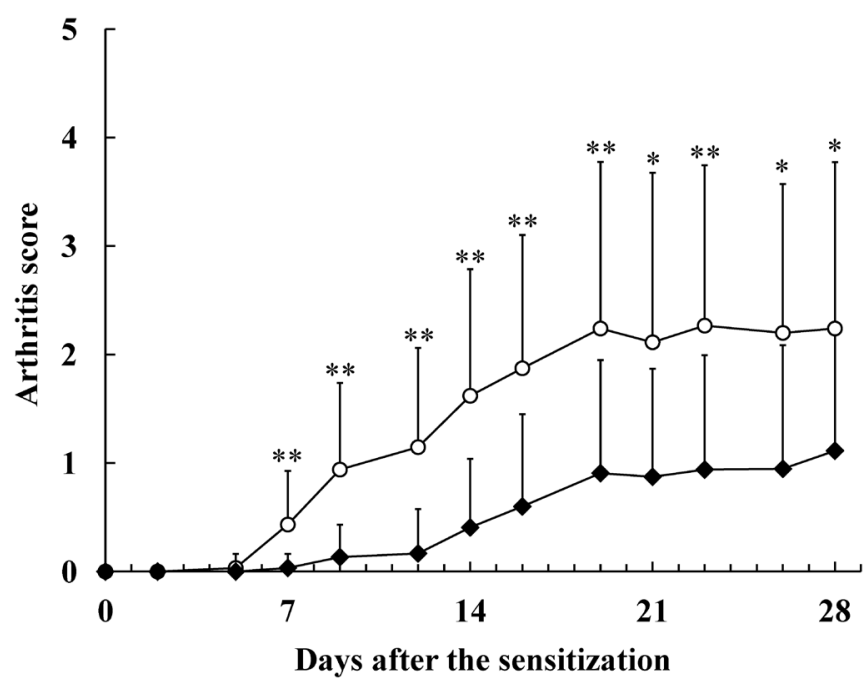

Fig. 2. Influence of INFJ on Arthritic Scores in SKG Mice

SKG mice were given $50 \%$ (v/v) INFJ (closed diamond) while the control group received drinking water (open circle). Each value was the mean \pm S.D. $(n=15)$. $*: p<0.05$ and **: $p<0.01 v s$. the control group using the $t$-test. Arthritic scores were significantly lower in the INFJ group than the control group from day 7 after the Mannan sensitization.

Table 1. Histopathology Scores of SKG Mice

\begin{tabular}{lcccc}
\hline \hline Group & Synovium & Inflammation & Pannus formation & Total score \\
\hline Control & $9.17 \pm 1.33$ & $9.83 \pm 2.64$ & $10.5 \pm 2.0$ & $29.5 \pm 5.4$ \\
INFJ & $7.17 \pm 2.23$ & $5.67 \pm 2.66$ & $7.7 \pm 2.2$ & $20.5 \pm 6.6$ \\
$p$ Value & $p=0.088$ & $p<0.05$ & $p<0.05$ & $p<0.05$ \\
\hline
\end{tabular}

Each value was the mean \pm S.D. $(n=6)$. 

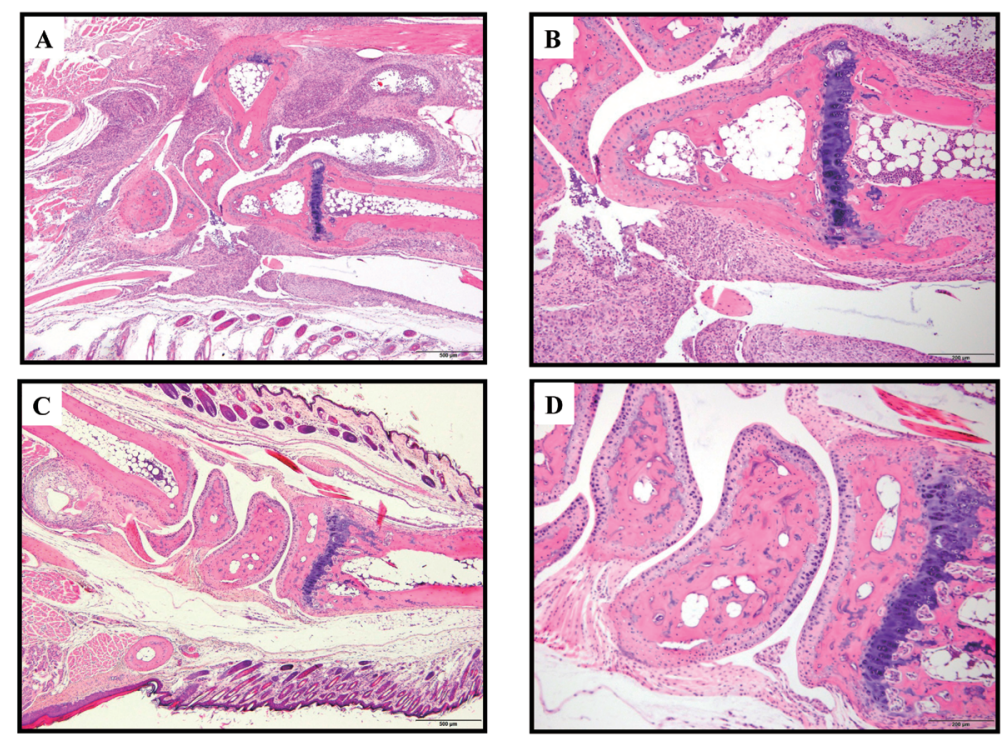

Fig. 3. Influence of INFJ on the Histopathologies of Wrist and Ankle Joints in SKG Mice

Marked changes in synoviocyte proliferation with inflammation and pannus formation were observed in the control group (original magnification, A and magnification $40 \times, \mathrm{B}$; respectively), but not in the INFJ group (original magnification, C and magnification 40×, D; respectively).

Table 2. Hematology

\begin{tabular}{llccccc}
\hline \hline & Group & $\begin{array}{c}\text { White blood cells } \\
(/ \mu \mathrm{L})\end{array}$ & $\begin{array}{c}\text { Red blood cells } \\
\left(\times 10^{3} / \mu \mathrm{L}\right)\end{array}$ & $\begin{array}{c}\text { Hemoglobin } \\
(\mathrm{g} / \mathrm{dL})\end{array}$ & $\begin{array}{c}\text { Hematocrit } \\
(\%)\end{array}$ & $\begin{array}{c}\text { Platelets } \\
\left(\times 10^{3} / \mu \mathrm{L}\right)\end{array}$ \\
\hline Sub-acute toxicity (28d) & Control & $6700 \pm 704$ & $10042 \pm 910$ & $17.2 \pm 0.7$ & $60.3 \pm 5.3$ & $1272 \pm 181$ \\
& INFJ & $6650 \pm 281$ & $9865 \pm 280$ & $17.6 \pm 0.5$ & $60.1 \pm 2.3$ & $1137 \pm 108$ \\
& $p$ Value & N.S. & N.S. & N.S. & N.S. & N.S. \\
Sub-chronic toxicity (90 d) $)$ & Control & $4433 \pm 1515$ & $10275 \pm 457$ & $17.7 \pm 0.5$ & $59.9 \pm 2.8$ & $1429 \pm 135$ \\
& INFJ & $4750 \pm 742$ & $10482 \pm 210$ & $17.7 \pm 0.3$ & $60.1 \pm 1.2$ & $1305 \pm 89$ \\
& $p$ Value & N.S. & N.S. & N.S. & N.S. & N.S. \\
\hline
\end{tabular}

Each value was the mean \pm S.D. $(n=6)$. not significant (N.S.).

drink. In this study, we aimed to examine anti-arthritic potential using SKG autoimmune animal model. After $7 \mathrm{~d}$ from mannan treatment, the arthritis was developed at both the ankles and wrists and its severerity became prominent along with the experiment period of $28 \mathrm{~d}$ in the control group. INFJ inhibited significantly in arthritic scores compared with the control group. Histopathological examination demonstrated the typical arthritic changes with inflammation, proliferation of synoviocytes and pannus formation. In the control group, neutrophil-rich exudates in the joint cavity were found in some cases. INFJ administration significantly suppressed the characteristic lesions of RA compared with the control group, though the grade of inflammatory cell infiltration was not statistically significant. In statistical analysis of total scores, histopathological grade of lesions was significantly lower in the group served with INFJ than that with water. The arthritic scores of the joints were almostly comparable with histopathological evaluation in inhibition of severerity of SKG mice arthritis.

The mechanism of the anti-arthritic effect by noni juice is not fully understood. Now, we are studying the influence of compounds isolated and identified from INFJ on signal transducer and activator of transcription (STAT) 3 and IL-6, which play important roles in inflammation pathways, including those in RA. Part of the compounds showed the suppression effect of STAT3 expression and IL-6 production in vitro.
Table 3. Liver and Renal Functions

\begin{tabular}{llcc}
\hline \hline & Group & ALT (IU/L) & BUN (mg/dL) \\
\hline Sub-acute toxicity (28d) & Control & $35.1 \pm 51.1$ & $22.2 \pm 6.6$ \\
& INFJ & $26.0 \pm 10.6$ & $27.7 \pm 5.0$ \\
& $p$ Value & N.S. & $p=0.050$ \\
Sub-chronic toxicity (90d) & Control & $22.3 \pm 9.1$ & $26.2 \pm 3.5$ \\
& INFJ & $26.2 \pm 3.5$ & $27.8 \pm 2.8$ \\
& $p$ Value & N.S. & N.S. \\
\hline
\end{tabular}

Each value was the mean \pm S.D. $(n=6-12)$. not significant (N.S.).

Therefore, we consider that some compounds contained in noni juice may indicate anti-RA effect. Currently, detailed mechanisms regarding noni extract compounds are evaluated in vitro and in vivo.

Noni fruit juice was served free access to SKG mice. Water intake was smaller in the INFJ group than the control group. This might be associated with poor palatability to INFJ that gives unique taste and odor associated with medium chain fatty acid-esters. ${ }^{8,9)}$ However, there was no significant change in body weight in both groups. This indicates that lower intake of INFJ may not affect in anti-arthritic actions.

There are a few cases of hepatotoxicity associated with noni fruit juice in Caucasean societies. ${ }^{24-26)}$ The INFJ which caused it was originated from French Polynesia and formulated with 
flavor and other fruit ingredients. Therefore, INFJ was exploratory evaluated its safety by 28 and $90 \mathrm{~d}$ repeated dose tests in $\mathrm{BALB} / \mathrm{c}$ mice in the same administration way as the arthritic study. All the parameters measured did not reveal adverse events in hematology and hepatic and renal functions.

This is the first study that INFJ was demonstrated effective against RA in SKG autoimmune animal model resembling human RA. The results indicate that INFJ may lead to be safe and effective RA therapy.

\section{CONCLUSION}

The effects of INFJ for RA were evaluated using autoimmune RA model. It is concluded that the INFJ significantly inhibited both arthritic and histopathological scores and was found safe in repeated dose toxicity studies. The identification of active ingredients in INFJ and elucidation of the mode of action for anti-arthritis actions remain for further investigations.

Acknowledgments This work was supported by JSPS Core-to-Core Program, B. Asia-Africa Science Platforms.

Conflict of Interest The authors declare no conflict of interest.

\section{REFERENCES}

1) Firestein GS. Evolving concepts of rheumatoid arthritis. Nature, 423, 356-361 (2003).

2) Scott DL, Wolfe F, Huizinga TWJ. Rheumatoid arthritis. Lancet, 376, 1094-1108 (2010).

3) Smolen JS, Aletaha D, Koeller M, Weisman MH, Emery P. New therapies for treatment rheumatoid arthritis. Lancet, 370, 1861-1874 (2007).

4) Kurkó J, Besenyei T, Laki J, Glant TT, Mikecz K, Szekanecz Z. Genetics of rheumatoid arthritis - a comprehensive review. Clin. Rev. Allergy Immunol., 45, 170-179 (2013).

5) Kaur A, Nain P, Nain J. Herbal plants used in treatment of rheumatoid arthritis: a review. Int. J. Pharm. Pharmac. Sci., 4, 44-57 (2012).

6) Ridley HN, The Flora of The Malay Peninsula. Vol. II-Gamopetalae, L. Reeve \& Co., Ltd., London, pp. 117-118 (1923).

7) Wang MY, West BJ, Jensen CJ, Nowicki D, Su C, Palu AK, Anderson G. Morinda citrifolia (Noni): a literature review and recent advances in Noni research. Acta Pharmacol. Sin., 23, 1127-1141 (2002).

8) Assi RA, Darwis Y, Abdulbaqi IM, Khan AA, Vuanghao L, Laghari MH. Morinda citrifolia (Noni): a comprehensive review on its industrial uses, pharmacological activities, and clinical trials. Arabian J. Chem., 10, 691-707 (2017)

9) Chan-Blanco Y, Vaillant F, Perez AM, Reynes M, Brillouet JM, Brat P. The noni fruit (Morinda citrifolia L.): a review of agricultural research, nutritional and therapeutic properties. J. Food Compos. Anal., 19, 645-654 (2006).

10) Ikeda $R$, Wada $M$, Nishigaki $T$, Nakashima $K$. Quantification of coumarin derivatives in Noni (Morinda citrifolia) and their contribution of quenching effect on reactive oxygen species. Food Chem., 113, 1169-1172 (2009).

11) Sakaguchi N, Takahashi $T$, Hata H, Nomura $T$, Tagami $T$, Yamazaki
S, Sakihama T, Matsutani T, Negishi I, Nakatsuru S, Sakaguchi S. Altered thymic T-cell selection due to a mutation of the ZAP-70 gene causes autoimmune arthritis in mice. Nature, 426, 454-460 (2003).

12) Keller KK, Lindgaard LM, Wogensen L, Dagnaes-Hansen F, Thomsen JS, Sakaguchi S, Stengaard-Pedersen K, Hauge EM. SKG arthritis as a model for evaluating therapies in rheumatoid arthritis with special focus on bone changes. Rheumatol. Int., 33, 1127-1133 (2013).

13) Yoshitomi H, Sakaguchi N, Kobayashi K, Brown GD, Tagami T, Sakihama T, Hirota K, Tanaka S, Nomura T, Miki I, Gordon S, Akira S, Nakamura T, Sakaguchi S. A role for fungal $\beta$-glucans and their receptor Dectin-1 in the induction of autoimmune arthritis in genetically susceptible mice. J. Exp. Med., 201, 949-960 (2005).

14) Hata H, Sakaguchi N, Yoshitomi H, Iwakura Y, Sekikawa K, Azuma Y, Kanai C, Moriizumi E, Nomura T, Nakamura T, Sakaguchi S. Distinct contribution of IL-6, TNF- $\alpha$, IL-1, and IL-10 to T-cell mediated spontaneous autoimmune arthritis in mice. J. Clin. Invest., 114, 582-588 (2004).

15) Kobayashi K, Suda T, Nan-ya K, Sakaguchi N, Sakaguchi S, Miki I. Cytokine production profile of splenocytes derived from zymosan A-treated SKG mice developing arthritis. Inflamm. Res., 55, 335-341 (2006)

16) Hashimoto M, Hirota $K$, Yoshitomi H, Maeda S, Teradaira S, Akizuki S, Prieto-Martin P, Nomura T, Sakaguchi N, Kohl J, Heyman B, Takahashi M, Fujita T, Mimori T, Sakaguchi S. Complement drives Th17 cell differentiation and triggers autoimmune arthritis. $J$. Exp. Med., 207, 1135-1143 (2007).

17) Krenn V, Morawietz L, Häupl T, Neidel J, Petersen I, König A. Grading of chronic synovitis - a histopathological grading system for molecular and diagnostic pathology. Pathol. Res. Pract., 198, 317-325 (2002).

18) McKoy ML, Thomas EA, Simon OR. Preliminary investigation of the anti-inflammatory properties of an aqueous extract from Morinda citrifolia (Noni). Proc. West. Pharmacol. Soc., 45, 76-78 (2002).

19) Akihisa T, Matsumoto K, Tokuda H, Yasukawa K, Seino K, Nakamoto K, Kuninaga H, Suzuki T, Kimura Y. Anti-inflammatory and potential cancer chemopreventive constituents of the fruits of Morinda citrifolia (Noni). J. Nat. Prod., 70, 754-757 (2007).

20) Basar S, Uhlenhut K, Hogger P, Schone F, Westendorf J. Analgesic and antiinflammatory activity of Morinda citrifolia L. (Noni) fruit. Phytother. Res., 24, 38-42 (2010).

21) Dussossoy E, Brat P, Bony E, Boudard F, Poucheret P, Mertz C, Giaimis J, Michel A. Characterization, anti-oxidative and antiinflammatory effects of Costa Rican noni juice (Morinda citrifolia L.). J. Ethnopharmacol., 133, 108-115 (2011).

22) Jethani B, Sharma RK, Sharma V, Jain SK. Role of inflammatory mediators in anti-inflammatory activity of noni (Morinda citrifolia) on acute inflammatory process in rats. Asian J. Pharm. Clin. Res., 4, 55-59 (2011).

23) Saraswathi CD, Prakash WS, Kunal PW. Anti-arthritic activity of Morinda citrifolia L. fruit juice in complete freund's adjuvant induced arthritic rats. J. Pharm. Res., 5, 1236-1239 (2012).

24) Stadlbauer V, Fickert P, Lackner C, Schmerlaib J, Krisper P, Trauner M, Stauber RE. Hepatotoxicity of NONI juice: report of two cases. World J. Gastroenterol., 11, 4758-4760 (2005).

25) Millonig G, Stadlmann S, Vogel W. Herbal hepatotoxicity: acute hepattis caused by a Noni preparation (Morinda citrifolia). Eur. J. Gastroenterol. Hepatol., 17, 445-447 (2005).

26) Yüce B, Gulberg V, Diebold J, Gerbes AL. Hepatitis induced by Noni juice from Morinda citrifolia: a rare cause of hepatotoxicity of the tip of the iceberg? Digestion, 73, 167-170 (2006). 